\title{
Malignant Peripheral Nerve Sheath Tumor with Mesenchymal Differentiation
}

National Cancer Institute

\section{Source}

National Cancer Institute. Malignant Peripheral Nerve Sheath Tumor with Mesenchymal

Differentiation. NCI Thesaurus. Code C92647.

A malignant peripheral nerve sheath tumor characterized by the presence of mesenchymal differentiation. Representative example is the malignant Triton tumor which contains a rhabdomyosarcomatous component. 